\section{PTU-002 HYPERTENSION IS AN INDEPENDENT RISK FACTOR FOR COLORECTAL POLYPS}

doi:10.1136/gut.2011.239301.130

L Bell , * A Johnston, S MacKenzie, K Sundaram, J Mawdsley , I G Beveridge, C Collins, K J Monahan Department of Gastroenterology, West Middlesex University Hospital, London, UK

Introduction Components of metabolic syndrome including obesity and insulin resistance with colorectal neoplasia have been well characterised, but the association of other cardiovascular risk factors including hypertension have not been well defined. The aim of this study is to evaluate the association of other cardiovascular risk factors, and colonic polyp risk.

Methods A study of 1038 patients' colonoscopies performed between January 2009 and January 2010 was undertaken, recording polyp number, appearance and anatomical location. The risk factors evaluated in the study were as follows, hypertension, hyperlipidaemia, renal function and insulin resistance. The prospectively collected data was derived from colonoscopy reports, electronic medical records and serum blood sample results. Continuous variables were analysed using a $\chi^{2}$ test, and risk was evaluated using odds ratios.

Results A total of 1038 patients were included in the study, $32 \%$ (331 patients) were found to have one or more colonic polyp, of these patients $39 \%$ (131 patients) had co-existing hypertension compared to $26 \%$ of the patients without colonic polyps ( $\mathrm{p}$ valve $=0.000005 \chi^{2}$ test; OR $1.8995 \% \mathrm{CI}$ 1.43 to 2.49$)$. $77 \%$ of polyps were left sided. Upper and lower most quartiles for serum creatinine results showed an association with renal failure and the presence of colonic polyps. The upper most quartile for serum, creatinine showed a $60 \%$ association with colonic polyps while the lowest quartile had an association of $48 \%$ (p value $0.015 ; \chi^{2}$ test). Other risk factors did not demonstrate association with risk.

Conclusion Hypertension is an independent risk factor for colorectal polyps in this study. There is also an association of renal impairment with the development of polyps. The

Table 1 PTU-002 Results table of co-morbidities and association with colorectal polyps

\begin{tabular}{llllll}
\hline Variable & No polyps & Polyps & p Value $\chi \mathbf{2}$ & OR & $\mathbf{9 5 \% ~ C I ~}$ \\
\hline Total & 706 & 332 & - & - & - \\
Hypertension & 181 & 131 & 0.000005 & 1.89 & 1.43 to 2.49 \\
Diabetes & 68 & 41 & 0.18 & 1.32 & 0.88 to 2.0 \\
Hyperlipidaemia & 147 & 84 & 0.106 & 1.29 & 0.95 to 1.75 \\
\hline
\end{tabular}

mechanism may be related to metabolic syndrome or may have an independent aetiological pathway.

Competing interests None.

Keywords colonic polyps, colonoscopy, colorectal, hypertension.

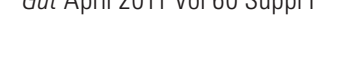

